\title{
Explicit solvation modulates intra- and inter-molecular interactions within DNA: Electronic aspects revealed by the $a b$ initio fragment molecular orbital (FMO) method
}

\author{
Kaori Fukuzawa $^{\mathrm{a}, \mathrm{b}, *}$, Ikuo Kurisaki ${ }^{\mathrm{c}}$, Chiduru Watanabe ${ }^{\mathrm{b}}$, Yoshio Okiyama ${ }^{\mathrm{b}}$, Yuji Mochizuki ${ }^{\mathrm{b}, \mathrm{d}}$, \\ Shigenori Tanaka ${ }^{e}$, Yuto Komeiji ${ }^{\mathrm{d}, \mathrm{f}, *}$ \\ a School of Dentistry at Matsudo, Nihon University, 2-870-1 Sakaecho-Nishi, Matsudo, Chiba 271-8587, Japan \\ ${ }^{\mathrm{b}}$ Institute of Industrial Science, The University of Tokyo, 4-6-1 Komaba, Meguro-ku, Tokyo 153-8505, Japan \\ ${ }^{c}$ Graduate School of Information Science, Nagoya University, Furo-cho, Chikusa-ku, Nagoya, Aichi 464-8601, Japan \\ ${ }^{\mathrm{d}}$ Department of Chemistry and Research Center for Smart Molecules, Faculty of Science, Rikkyo University, 3-34-1 Nishi-ikebukuro, Toshima-ku, Tokyo 171-8501, Japan \\ e Graduate School of System Informatics, Kobe University, 1-1 Rokkodai, Nada-ku, Kobe, Hyogo 657-8501, Japan \\ f Biomedical Research Institute, AIST Tsukuba Central 2, Tsukuba, Ibaraki 305-8568, Japan
}

\section{A R T I C L E I N F O}

Article history:

Received 5 October 2014

Received in revised form 26 November 2014

Accepted 27 November 2014

Available online 4 December 2014

\section{Keywords:}

Fragment molecular orbital method

DNA

Solvation

Interaction energy

Energy decomposition analysis

ABINIT-MP

\begin{abstract}
A B S T R A C T
The change in the electronic structure of a DNA duplex d(CGCGAATTCGCG $)_{2}$ upon solvation was investigated by the $a b$ initio fragment molecular orbital (FMO) method. The crystal structure of the duplex was immersed in a solvent box containing explicit water and $\mathrm{Na}^{+}$ions, and the resultant solvated DNA was relaxed and annealed by the classical molecular dynamics method. From the annealed structure a series of solvated DNA configurations were constructed with varying solvent shell thicknesses $(0-12 \AA)$. Each configuration was subjected to FMO calculation at the MP2/6-31G* level. Partial charge, internal energies, interaction energies between the bases and phosphate backbones, and fragment molecular orbitals within DNA were calculated and expressed as functions of the solvent thickness. Most of these physical properties within DNA converged at a shell thickness of $8 \AA$, indicating the dominant effect of the first and second solvation layers. Ca. $-7 e$ charge, i.e. $-0.6 e$ per base pair, was transferred from DNA to the solvent. Upon solvation the Watson-Crick H-bonds became stabilized but the stacking interactions were destabilized. Based on the pair interaction energy decomposition analysis, these stability changes were attributed to modulation of the electrostatic interaction elicited by the rearrangement of the charge distribution due to the charge transfer to the solvent. Thus, this study revealed significant modulation of the electronic structure of the DNA upon solvation and its impact on molecular interactions, which can be described only through quantum-chemical calculations.
\end{abstract}

() 2014 Elsevier B.V. All rights reserved.

\section{Introduction}

The physico-chemical nature of DNA has long attracted the interest of computational scientists. Solvated DNA in particular has been a topic favored by the classical force-field molecular dynamics (MD) simulations for the last two decades. Current state-of-the-art classical MD simulations of DNA are performed in a realistic environment made up of explicit ions and water molecules and have provided

\footnotetext{
* Corresponding authors at: School of Dentistry at Matsudo, Nihon University, 2 870-1 Sakaecho-Nishi, Matsudo, Chiba 271-8587, Japan. Tel./fax: +81 473609316 (K. Fukuzawa). Biomedical Research Institute, AIST Tsukuba Central 2, Tsukuba, Ibaraki 305-8568, Japan. Tel.: + 81298612447 (Y. Komeiji).

E-mail addresses: fukuzawa.kaori@nihon-u.ac.jp (K. Fukuzawa),y-komeiji@aist. go.jp (Y. Komeiji).
}

important insights into the conformational dynamics of the molecules (see [1] for review). Quantum-chemical calculations of DNA also have a long history [2], although for many years these calculations were performed in vacuo, and mostly only for small parts of DNA molecules (for example, studies of base-base interactions $[3,4])$. Thus it has only been in the past decade that quantum-chemical calculations of fully solvated DNA molecules have emerged. The semi-empirical study by Westerhoff and Merz [5] was the most important and extensive investigation in the quantum-chemical studies of DNA up to the mid-2000s, and described the modulation of the electronic properties of a DNA duplex upon solvation. The important findings of this study were as follows: ca. $-0.3 e$ or $-0.5 e$ per base pair is transferred from the DNA to the solvent; the band gap in the DNA is $\sim 1 \mathrm{eV}(\sim 20 \mathrm{kcal} / \mathrm{mol})$ without solvent but becomes ca. $2-3 \mathrm{eV}(\sim 50 \mathrm{kcal} / \mathrm{mol})$ upon solvation; and implicit 
solvent models can usually but not always mimic the explicit solvent. A preceding density functional theory (DFT) calculation on a solvated DNA [6] reported a narrower band gap value of $1.28 \mathrm{eV}$ ( $30.2 \mathrm{kcal} / \mathrm{mol})$. The difference was presumably attributable to the difference in the calculation methods. Thus, the classical and semiempirical quantum calculations have provided important insights into the DNA solvation. In this study, we attempt to further clarify the DNA solvation using $a b$ initio quantum-chemical calculations.

For many years, the large size of explicitly solvated DNA prevented its $a b$ initio quantum-chemical calculations (ca. 10,000 atoms [2]), but the advent of the fragment molecular orbital method (FMO, [7-9]) changed this situation. In the FMO method, the molecular system of interest is divided into fragments, and then each fragment and fragment pair is subjected to self-consistent field (SCF) calculations under environmental electrostatic potentials, and the electronic structure of the whole system is reconstructed. This procedure reduces the computational cost of the Hartree-Fock (HF) calculations from $O\left(N^{3}\right)$ to $O\left(N^{2}\right)$ or less, is easy to parallelize, and enables the inter-fragment interaction energy analysis (IFIE, [10-12]; also called the pair interaction energy, PIE [13]). FMO has been extended from HF to the secondorder Møeller-Plesset (MP2) method and DFT to include the electron correlation effect. Several FMO calculations have been performed for molecular systems involving DNA or RNA [14-18]. In addition, we recently reported a series of FMO benchmark calculations of one or two-base DNA molecules, single or double stranded, and with or without counterions [19], which revealed the necessity of the charge neutralization, the importance of appropriate fragmentation, and the degree of dependence of IFIE upon neutralization. In the current report we try to extend this in vacuo benchmark to solvated DNA, which has become all the more important along with the discovery of FMO as an advantageous method for incorporating an explicit solvent at a reasonable computational cost $[14,20]$.

Here, we report benchmark FMO calculations of DNA in a realistic solvent, where "solvent" refers to explicit ions and water as an entity. We designed this study along the lines of similar previous studies $[5,20]$. In brief, we started with a crystal structure of the Dickerson dodecamer, d(CGCGAATTCGCG) $)_{2}$, the same sequence studied in Ref. [5], solvated the structure with explicit water and $\mathrm{Na}^{+}$ions, performed classical MD to relax and anneal the solvated structure, and constructed a series of B-form DNA configurations with differently sized solvent shells from the annealed structure. Then the solvated DNA configurations were subjected to FMO electronic structure calculations to obtain several physical quantities within the DNA as functions of the solvent size, the physical properties including such as intra-molecular energy, atomic charge, and molecular orbitals (MOs). The effects of the solvent on the DNA are discussed to establish general guidelines for the electronic structure calculations of DNA under a realistic environment.

\section{Methods}

A series of configurations of solvated DNA were constructed with a classical force field method and were subjected to FMO calculations.

\subsection{Preparation of solvated DNA}

The molecular configurations for the FMO calculations were prepared as follows. The 3D crystal structure of the Dickerson dodecamer ([21], PDB entry: 355D) was immersed in a box of explicit water molecules containing $22 \mathrm{Na}^{+}$ions, enough to neutralize the DNA charge. The DNA/solvent system was annealed by classical molecular dynamics and energy minimization using
PEACH ver. 7.5 [22,23] with the AMBER99 force field [24] and TIP3P water model [25]. Among the resulting annealed structures, one annealed from the 0.9 ns snapshot was judged by the 3DNA analytical program [26] to have best retained the B-type conformation, and therefore was chosen as the template to construct a series of solvated DNA configurations with solvent shells of various thicknesses $(0,3,4,5, \ldots$, and $12 \AA$; Table $\mathrm{Si1}-1)$. $\mathrm{An} \mathrm{Na}^{+}$ion or a water molecule was regarded to reside in the shell if its distance from the nearest DNA atom was smaller than the specified thickness, the distance being measured considering only heavy atoms and by neglecting hydrogens. The series of configurations thus prepared were used in all the FMO calculations (Fig. 1). See Si1 for details.

\subsection{FMO calculations}

All the FMO calculations were performed using the ABINIT-MP program $[9,27,28]$ to the two-body expansion (FMO2) at the MP2/6-31G*level [29-31]. A nucleotide unit of DNA was appropriately divided into base and phosphate fragments (Fig. 2, designated as option 1 in Ref. [19]). An $\mathrm{Na}^{+}$ion and water molecules within $2.7 \AA$ of the ion were merged into a fragment, while each of the remaining water molecules was regarded as a separate fragment. Some of the data were calculated by multiple versions of ABINITMP to confirm the reproducibility of the data and the downward compatibility of the program.

\subsection{Analyses of the FMO results}

Interaction energies within the DNA/solvent system were calculated based upon IFIE, in a manner similar to that in a previous report [20]. By FMO2 the total energy of the system $(E)$ is obtained as the sum of effective internal-fragment energies $\left(E_{I}^{\prime}, I=1, \ldots, N\right)$ and inter-fragment energies $\left(\Delta E_{I J}, I<J\right)$ as follows:

$E=\sum_{I} E_{I}^{\prime}+\sum_{I<J} \Delta E_{I J}$

Let $A$ be a partial group of the fragments in the molecular complex. The effective internal energy within group $A\left(E_{A}\right)$ is then obtained by summation of $E_{I}^{\prime}$ and $\Delta E_{I J}$ :

$E_{A}=\sum_{I \in A} E_{I}^{\prime}+\sum_{(I<J) \in A} \Delta E_{I J}$
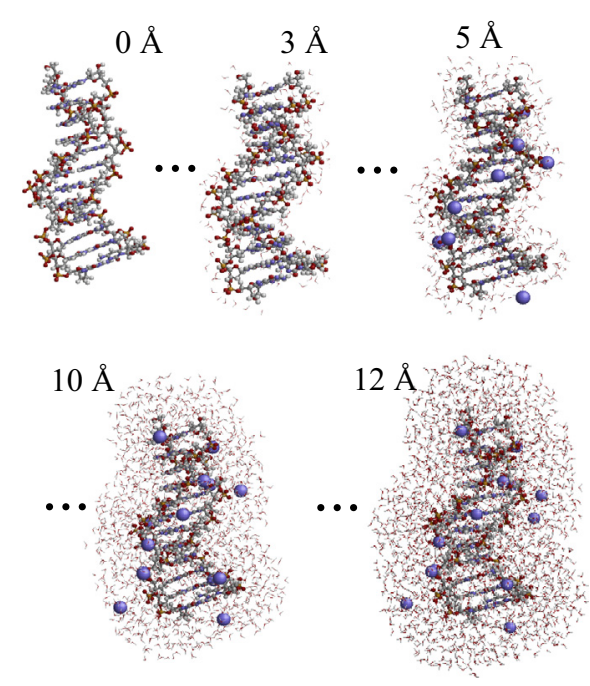

Fig. 1. Examples of configurations of the DNA/solvent complex with different solvent thicknesses. The purple spheres represent the $\mathrm{Na}^{+}$ions. See Table Si1-2 for data related to the size of the configurations. 


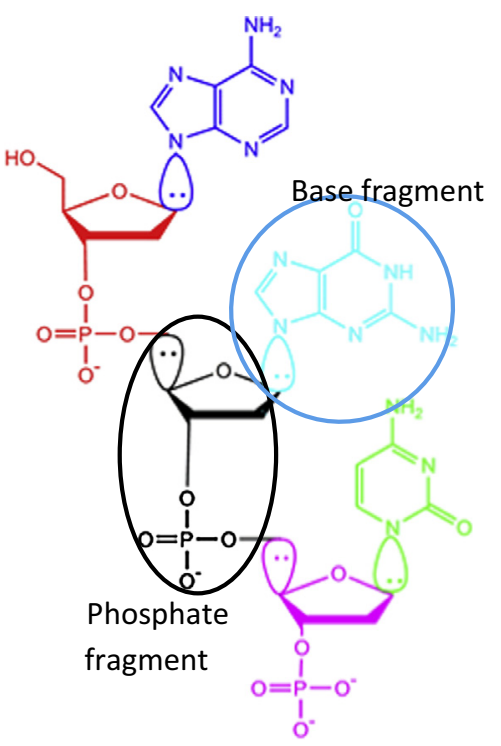

Fig. 2. The fragmentation scheme of DNA employed in this study.

The interaction energy of $A$ with another group $B\left(\Delta E_{A B}\right)$ is calculated as:

$\Delta E_{A B}=\sum_{I \in A} \sum_{J \in B} \Delta E_{I J}$

Groups $A$ and $B$ can be any pair of clusters of fragments. The IFIEs between base fragments (Fig. 2) were regarded as the Watson-Crick (W-C) H-bonding and stacking interactions (Fig. 3).

The atomic charges were calculated by the natural population analysis method (NPA [32,33]). An IFIE value was further decomposed into electrostatic (ES), exchange repulsion (EX), charge transfer with higher order mixed terms (CT + mix), and dispersion (DI) contributions by the pair interaction energy decomposition analysis (PIEDA [13]) implemented in the MIZUHO/BioStation3.0 program [34].

\section{Results and discussions}

We present below the overall effect of solvation on DNA (3.1) and then discuss the modulation of interactions within DNA upon solvation (3.2).

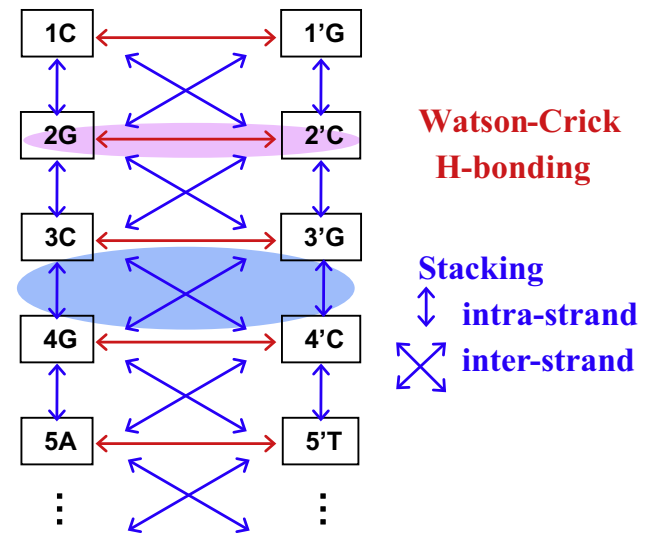

Fig. 3. Definition of base-base interactions within the double strand DNA. A W-C $\mathrm{H}$-bond (shown in red) is formed between two bases belonging to different strands. Stacking interactions (blue) refer to that between two bases belonging to the same strand (intra-strand stacking) and that between two bases belonging to different strands (inter-strand stacking). (For interpretation of the references to color in this figure legend, the reader is referred to the web version of this article.)

\subsection{Overall effect of solvation on DNA}

We estimated the internal energy, net charge, interaction energies, and frontier orbital energies of a fragment monomer of the DNA portion of the solvated complex as functions of the solvent thickness ( $0-12 \AA)$ to examine the overall effect of solvation and also to determine a solvent size small enough to converge these physical properties. Some care should be taken in interpreting the data because all the data were obtained in the presence of a negative charge, since the number of $\mathrm{Na}^{+}$ions included in the solvent varied depending on the shell thickness (Table Si1-2).

\section{(1) Internal-DNA energy}

The internal-DNA energy (Eq. (2)) rose, i.e., destabilized, along with the increase of the solvent size and converged at around $8 \AA$ (Fig. 4). The destabilization of the internal energy is a general phenomenon that occurs irrespective of the type of molecule of interest [20], provided that the molecular structure, i.e., the spatial arrangement of the nuclei, is invariant in the configurations investigated. Namely, in the absence of the solvent (thickness = $0 \AA$ ) the electrons optimize themselves solely to DNA while with the solvent they reoptimize their state to the solvated environment, thereby destabilizing the internal-DNA energy. The convergence around $8 \AA$ indicates the dominant influence of the first and second solvation layers on the internal-DNA energy.

\section{(2) NPA charge}

The NPA charge suggests that the effect of the charge transfer from DNA is dominantly governed by the water in direct contact with DNA-namely, the first solvent layer. The NPA charges of the DNA, water, $\mathrm{Na}^{+}$ions, and their summation (net) were separately plotted as functions of the solvent shell thickness (Fig. 5). Recall that each solvent shell contained at most $15 \mathrm{Na}^{+}$ions (Table Si1-2), which partially neutralized the DNA charge of $-22 e$. This partial neutralization resulted in the net charge of $-7 e$ at $12 \AA$. The charge of DNA went from $-22 e$ in vacuo $(0 \AA)$ to $-16 e$ at $3 \AA$ and converged at $-15 e$ at $8 \AA$. This converged DNA charge corresponds to a charge transfer of $-7 e$ in total, or ca. $-0.6 e$ per base pair, to the solvent. The charge transfer was marginally established even at the thinnest water shell of $3 \AA$ containing no $\mathrm{Na}^{+}$and was fairly invariant regardless of the number of water molecules, the number of ions, or the net charge of the system. This observation implies that the first solvation layer dominantly determined the amount of charge transfer from DNA. The charge was mostly transferred from the phosphate

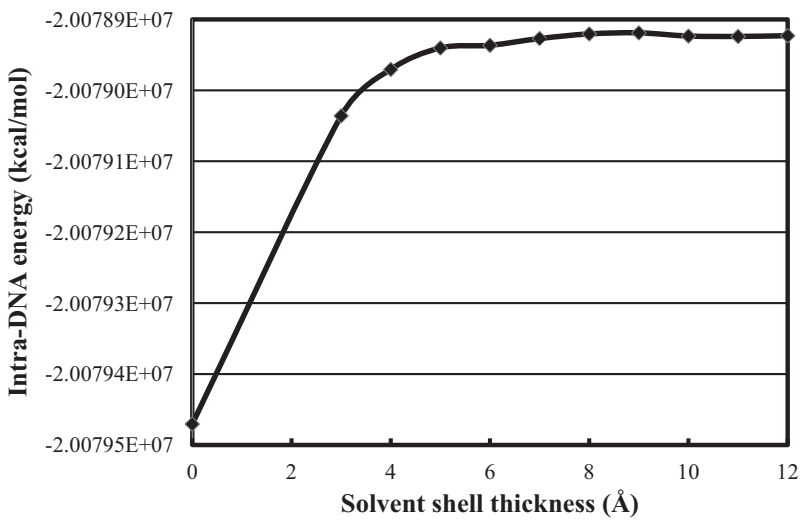

Fig. 4. Internal-DNA energy as a function of the solvent thickness calculated at $\mathrm{MP} 2 / 6-31 \mathrm{G} *$. 


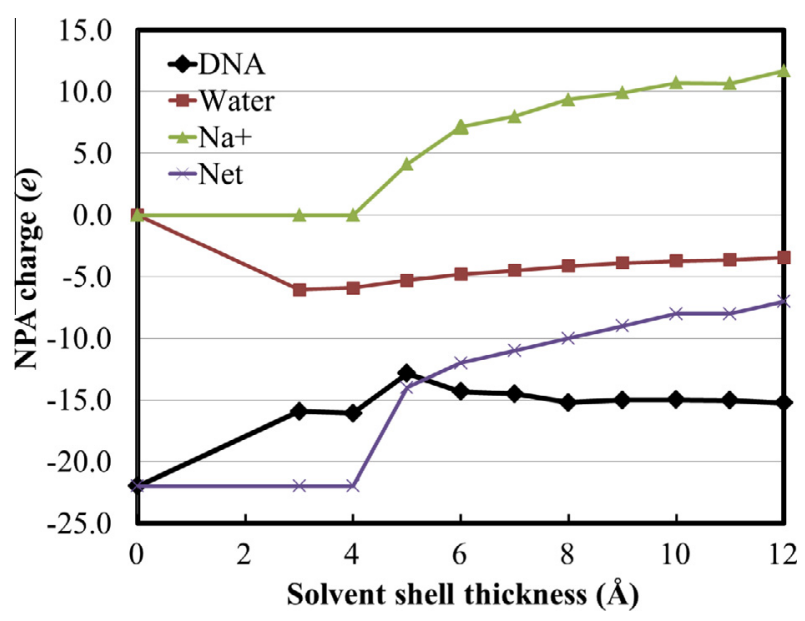

Fig. 5. NPA charge of each subsystem of the solvated DNA. Note that the "net charge" depends directly on the number of $\mathrm{Na}^{+}$ions contained in the solvent shell. For example, the net charge of $-22 e$ at $0 \AA$ corresponds to the bare charge of the DNA, but at $5 \AA$ it is partially neutralized to $-14 e$ by eight $\mathrm{Na}^{+}$ions.

fragments rather than the base fragments, as is clearly shown by the Qs-Qv values in Table Si2-3: the phosphate fragments showed a transfer of ca. $+0.25 e$ while the charge transfer of the base fragments was only ca. $+0.05 e$.

The charge transfer presented above agreed qualitatively, if not quantitatively, with the semi-empirical data of Westerhoff and Merz [5]. They performed both AM1 and PM3 calculations, with the former estimating the charge transfer per base pair at $-0.3 e$ and the latter at $-0.5 e$. The PM3 value was closer to ours, $-0.6 e$. Though these values quantitatively disagreed with each other, the disagreement should be within the range acceptable error because the partial charge was subject to change due to the type of quantum calculations used, and also due to the definition of the charge. On the whole, both their data and our present findings present a general picture in which the DNA, mainly the phosphate backbone, transfers some amount of negative charge to the solvent, with the quantity of the charge being governed by the first solvent layer.

\section{(3) IFIE}

The IFIE analysis revealed some interesting influences of the solvent on the base-base interactions. The charts of the $\mathrm{W}-\mathrm{C} \mathrm{H}$ bond energies, notably those of CG pairs but also those of AT pairs, went down upon solvation and converged at a solvent thickness of ca. 4-8 A (Fig. 6A), showing that the solvent stabilized the W-C Hbonds. In contrast to the $\mathrm{W}-\mathrm{C} \mathrm{H}$-bonds, the stacking energies within DNA rose slightly along with the size of the solvent and became fairly invariant after $4 \AA$ (Fig. 6B), revealing their solventdependent destabilization. The degree of destabilization varied among fragment pairs, but the overall trend was a few $\mathrm{kcal} / \mathrm{mol}$ per each pair. We will discuss these different behaviors of the base-base interactions in Section 3.2, and conclude here only that a solvent as thick as $8 \AA$ suffices for the convergence of both the $\mathrm{W}-\mathrm{C} \mathrm{H}$-bonds and stacking interactions.

\section{(4) Frontier MO energies}

The orbital energies of the frontier orbitals of the fragments provided further evidence of the necessity and sufficiency of a solvent shell as thick as $8 \AA$. The orbital energies, namely HOMO, LUMO and HOMO-LUMO gap energies, were averaged over each fragment species (Fig. 2 base or phosphate) and plotted as
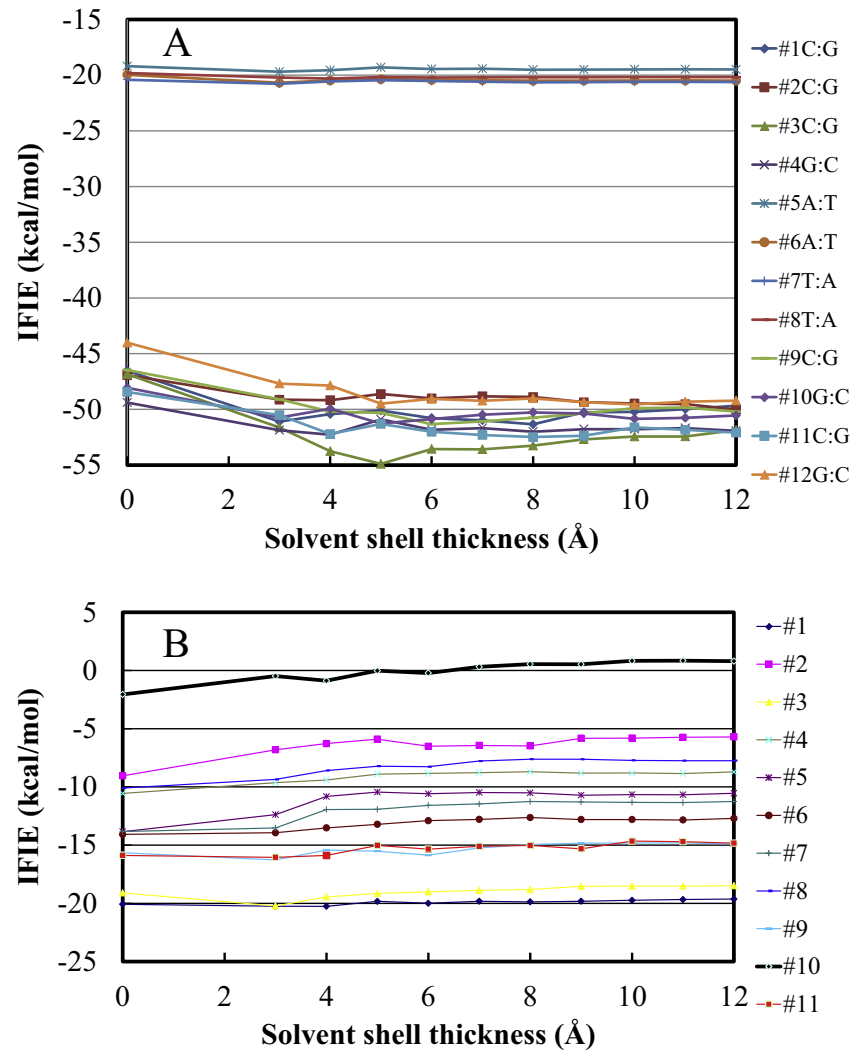

Fig. 6. Base-base interactions (IFIEs) as functions of the solvent thickness. (A) Watson-Crick H-bond interactions. (B) Stacking interactions. Pair \#1 refers to $1 \mathrm{C}: \mathrm{G}$ 2C:G stacking, \#2 to $2 \mathrm{C}: \mathrm{G} / 3 \mathrm{C}: \mathrm{G}, \ldots$, and \#11 to $11 \mathrm{C}: \mathrm{G} / 12 \mathrm{C}: \mathrm{G}$.

functions of the solvent thickness (Fig. 7). The HOMO energies of all the fragment species became negative when the solvent was thicker than $8 \AA$ (Fig. 7A), indicating the critical contribution of the first and second solvent layers for the stable existence of the DNA. The LUMO energies showed a similar solvent dependence (Fig. 7B), but generally the difference between HOMO and LUMO, namely HOMO-LUMO gap, is more important. The HOMO-LUMO gap of each fragment species increased with the solvent size, but became stable after ca. $6 \AA$ (Fig. 7C); this tendency was more prominent with the phosphate fragments than with the base fragments (Fig. 7D). This solvent-dependent increase of the HOMO-LUMO gap may reflect the general view that DNA is semi-conductive in vacuo but non-conductive in solvent [5]. Generally in HF MO calculation, however, the energy values of LUMO and consequently the HOMOLUMO gap are only qualitative [35]. Also, the frontier MO energies in Fig. 7 are those of the fragments, not that of the whole DNA. Thus, we suggest only that solvent could confer some non-conductivity onto the DNA by changing the electronic state of the phosphate backbone. We have briefly shown here that the solvent should be at least $8 \AA$ thick or larger for the frontier MO energies of the fragments to converge.

\section{(5) Summary of the overall solvent effect on DNA}

Thus, the internal energy, partial charge, and base-base interactions in the DNA portion of the solvated complex showed a marginal convergence at $4 \AA$ and a more complete one at $8 \AA$. The HOMO energies nonetheless did not converge completely even with a shell as thick as $12 \AA$, but their values became negative at $8 \AA$ and decreased only moderately afterwards. Taken together, these results suggest that the electronic state of the solvated 

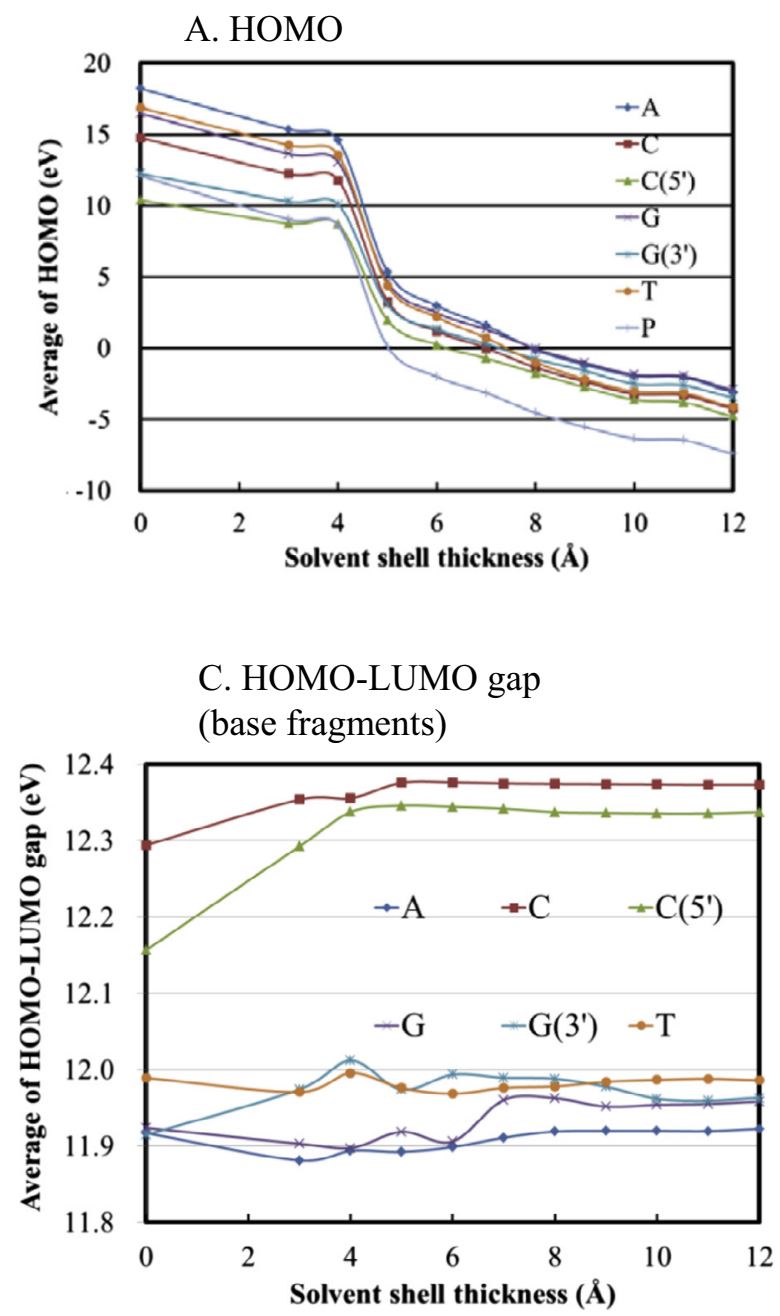

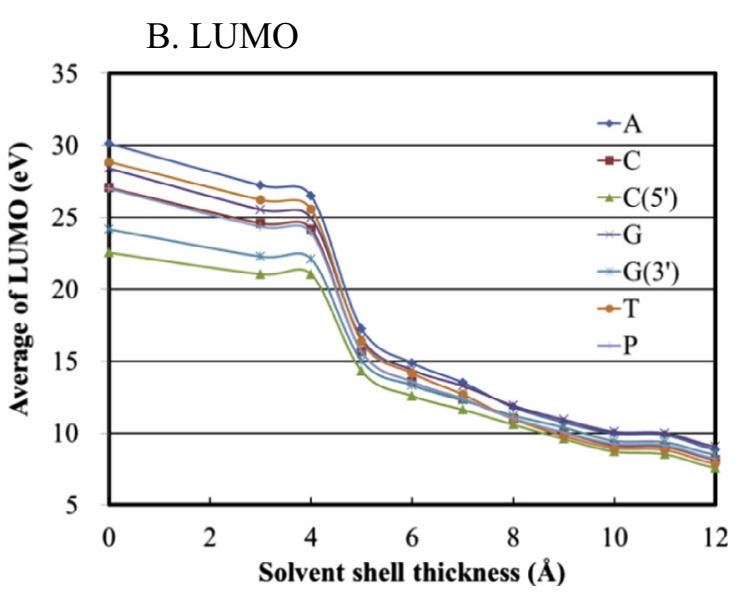

D. HOMO-LUMO gap

(phosphate fragments)

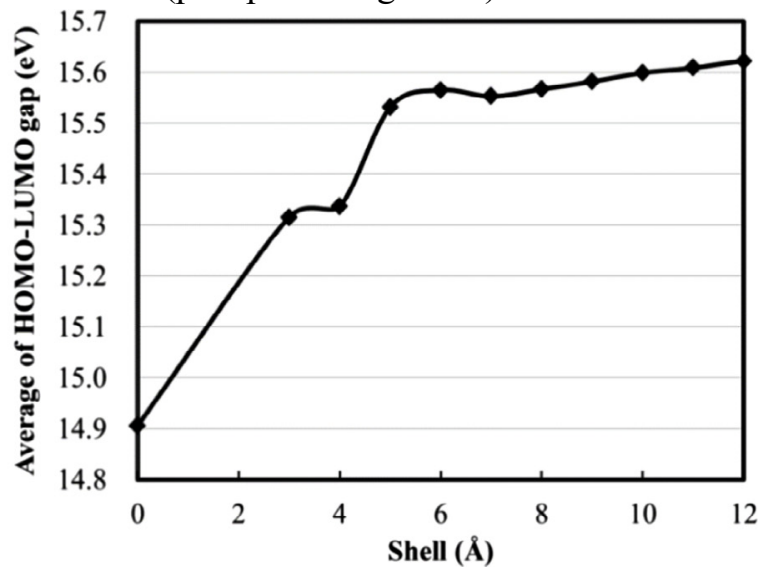

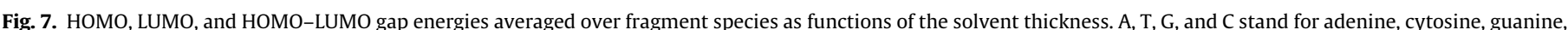

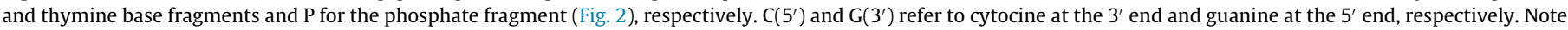

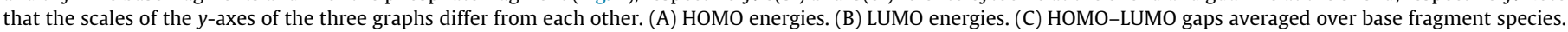
(D) HOMO-LUMO gap averaged over phosphate backbone fragments.

DNA is largely established with solvent molecules within 8 A of the surface, which roughly correspond to the first and second solvation layers.

We used FMO2 throughout this study in spite of the concern about the accuracy of FMO2 for the solvated $\mathrm{Na}^{+}$ions. The low accuracy of FMO2 was demonstrated by the importance of the three-body terms (FMO3) in atomic charge calculation of a hydrated $\mathrm{Na}^{+}$ion via the Mulliken population analysis (MPA) [36]. We nonetheless considered that inexpensive FMO2 should suffice for the present study because of the following reasons. Firstly, the NPA charge on a hydrated $\mathrm{Zn}(\mathrm{II})$ ion was essentially uninfluenced by the difference between FMO2 and FMO3, unlike the MPA charge [37]. Secondly, as no $\mathrm{Na}^{+}$ion was present within $4 \AA$ of the DNA (Table Si1-2, Fig. 1), the effect of the $\mathrm{Na}^{+}$ions on the electronic structure of the DNA should be rather indirect. Thirdly, most discussions in this study were focused on the fragment-fragment interactions based on the two-body terms and on the fragment molecular orbitals based on the single-body terms, where the effect of the three-body terms should be limited. Hence, the essential features of the electronic structure of DNA obtained here by FMO2 should likely hold even if FMO3 were used. The FMO3 calculation for solvated DNA systems remain as a future issue to be solved.
Considering the overall effect and adding a margin of safety, we chose the configuration with a solvent thickness of $10 \AA$ for minute analyses of the interactions within DNA presented in the following subsection.

\subsection{Modulation of the intra-DNA interactions via solvation}

Here we delve into the modulation of the interactions within DNA raised in the previous subsection, specifically the solvent-driven stabilization of the $\mathrm{W}-\mathrm{C}$ H-bond and destabilization of the stacking, by investigating the IFIE diagrams (Fig. 8), PIEDA (Fig. 9, Tables Si2-1 and Si2-2), and NPA (Fig. 10 and Table Si2-3). These data will show that the solvent induced a charge-redistribution within DNA and that the redistribution modulated the electrostatic contribution both to the $\mathrm{W}-\mathrm{C} \mathrm{H}$-bonding and stacking interactions to stabilize the former and destabilize the latter.

We will first discuss the stabilization of the $\mathrm{W}-\mathrm{C}$ H-bond interactions upon solvation (Fig. 6A and horizontal lines of Fig. 8). To understand the origin of the stabilization, the $\mathrm{W}-\mathrm{C}$ interactions were decomposed by PIEDA (Table Si2-1). The PIEDA indicated that the H-bond interaction comprised various components both in vacuo and in solvent, with ES being the largest and $\mathrm{CT}+\operatorname{mix}$ the second largest (Table Si2-1 A and B), but that their difference 


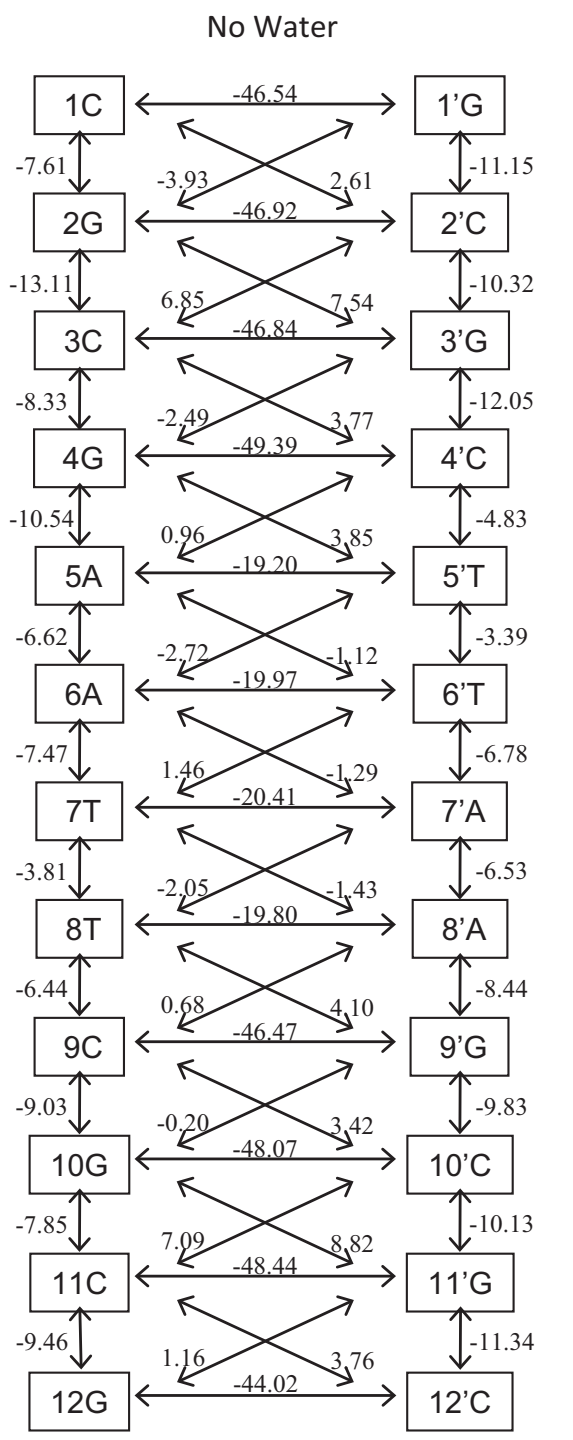

\begin{tabular}{c} 
1,2-stacking \\
energies \\
-20.1 \\
-9.0 \\
-19.1 \\
-10.5 \\
-13.9 \\
-15.9 \\
-10.1 \\
\hline-13.8
\end{tabular}

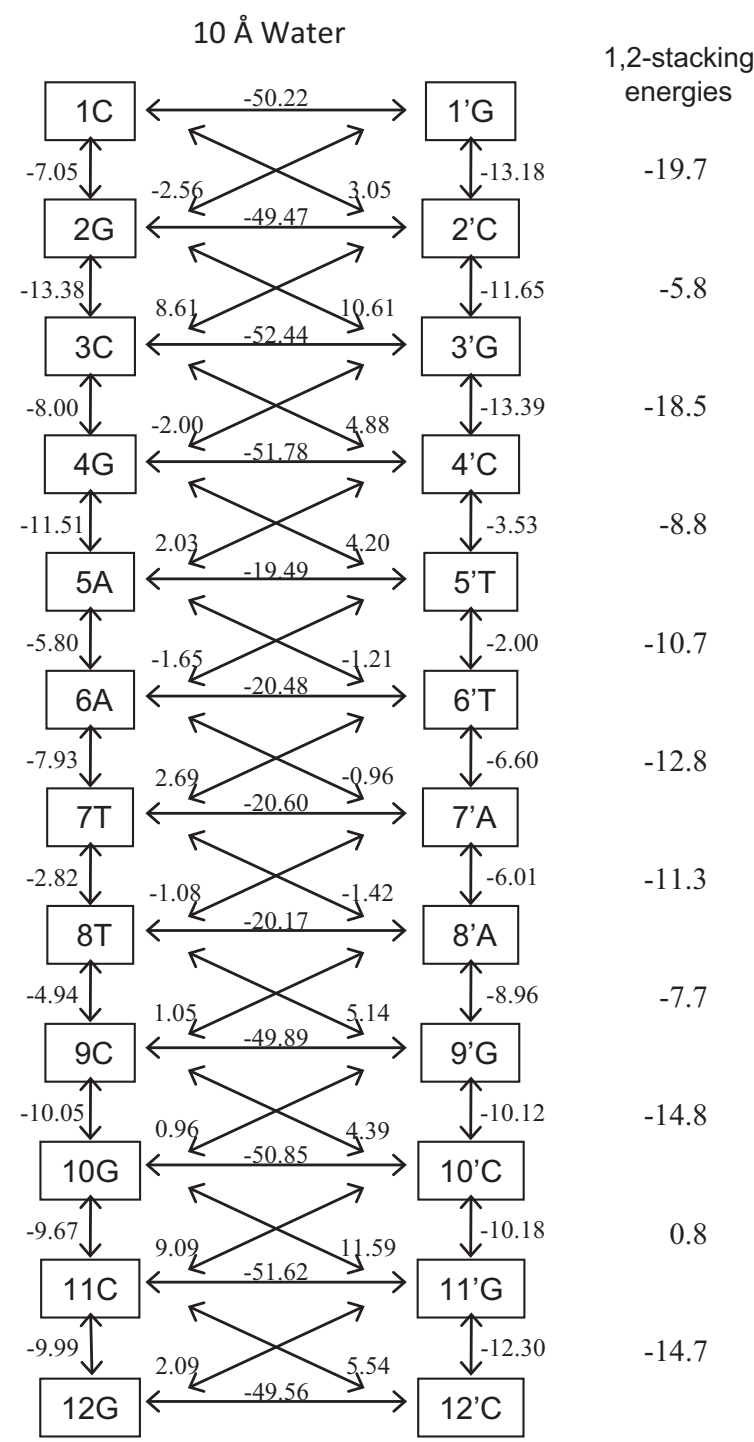

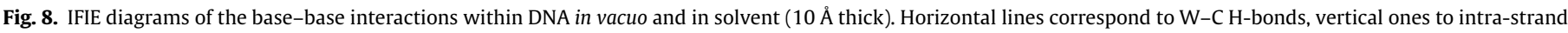

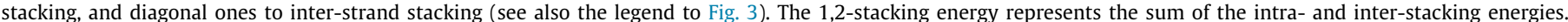
Energies are in $\mathrm{kcal} / \mathrm{mol}$.

consisted mostly of ES in all the base pairs (Table Si2-1C). The $\mathrm{CT}+\operatorname{mix}$ contributed destabilizing (positive) values, but this contribution was negligible compared to the large stabilizing (negative) contribution of ES. The change in the electrostatic interaction was therefore proven to be responsible for the solvent-dependent stabilization of the $\mathrm{W}-\mathrm{C}$ H-bonds.

The solvent-dependent stabilization of $\mathrm{W}-\mathrm{C} \mathrm{H}$-bond was more prominent for GC pairs (ca. $-3 \mathrm{kcal} / \mathrm{mol}$ per base pair) than for AT pairs (ca. $-0.4 \mathrm{kcal} / \mathrm{mol}$ per base pair). The difference in the number of $\mathrm{H}$-bonds within a pair (three in GC and two in AT) alone cannot explain the difference in the stabilization of the H-bonds. Rather, this difference between base pair species can be explained by the fact that the $\mathrm{G}$ bases tended to contribute more electrons to the solvent than other bases (Table Si2-3), presumably because guanine has the lowest ionization potential of the four bases [38]. Namely, a significant amount of negative charge was transferred from the $\mathrm{G}$ bases to the solvent, and this charge transfer should have relieved repulsion between the GC pairs to strengthen the $\mathrm{W}-\mathrm{C}$ H-bond. This explanation agrees well with the dominant contribution of ES to the solvent-dependent stabilization of the $\mathrm{W}-\mathrm{C}$ H-bond.

Next, we turn to the solvent-dependent destabilization of the stacking interactions. The IFIE diagram (Fig. 8) separately presents the intra- and inter-strand stacking energies and their summation (1,2-stacking energy). In contrast to the W-C H-bonding, the 1,2stacking energies became destabilized upon solvation by $0.4-$ $3.2 \mathrm{kcal} / \mathrm{mol}$. We also see that generally the intra-strand stacking was attractive but that the inter-strand stacking was repulsive. In solvent, the repulsive nature of the inter-strand stacking increased and resulted in the destabilization of the 1,2-stacking energies. In addition to the solvent effect, we found an important difference in the stacking interaction between our data and those in the literature. In our data many of the inter-strand stacking interactions took positive values (repulsive; Fig. 8 diagonal lines), while they were mostly negative (attractive) in Refs. $[3,4]$. This discrepancy is attributable to the partial charge of the base portion; namely, in our data each base portion carried a charge of ca. $+0.3 e$ (Table Si2-3) that presumably brought about the repulsion but in the previous molecular models no partial charge was assigned to the isolated base $[3,4]$. Therefore, the discrepancy suggests the importance of the realistic representation of the DNA molecule and surrounding environment in assessment of the interactions within DNA.

The stacking energies were further decomposed by PIEDA (Table Si2-2). Though the stacking energies consisted of various 
Total IFIE

ES

EX

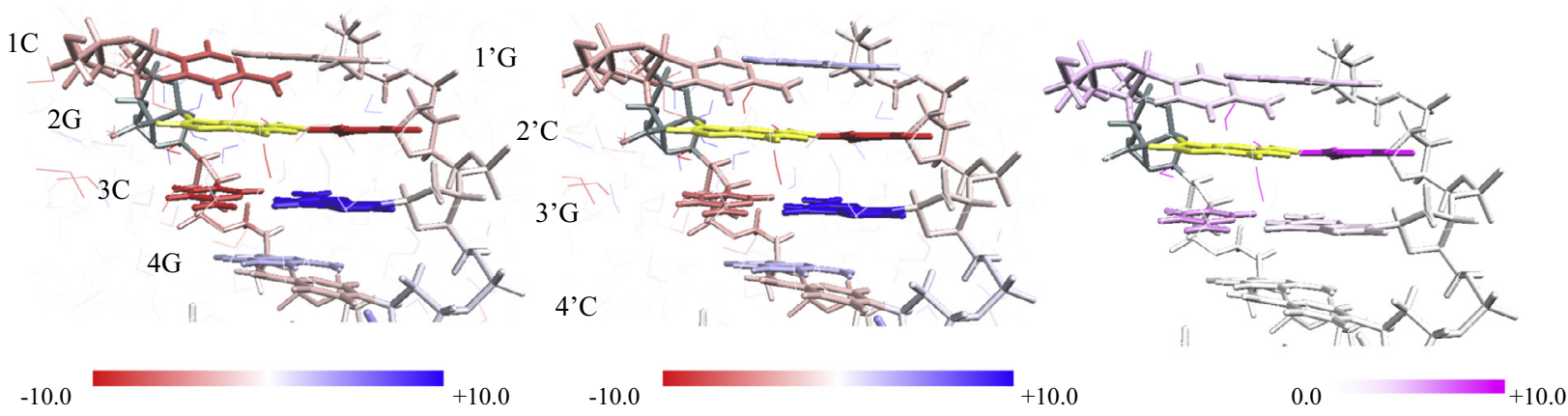

$\mathrm{CT}+\operatorname{mix}$

DI
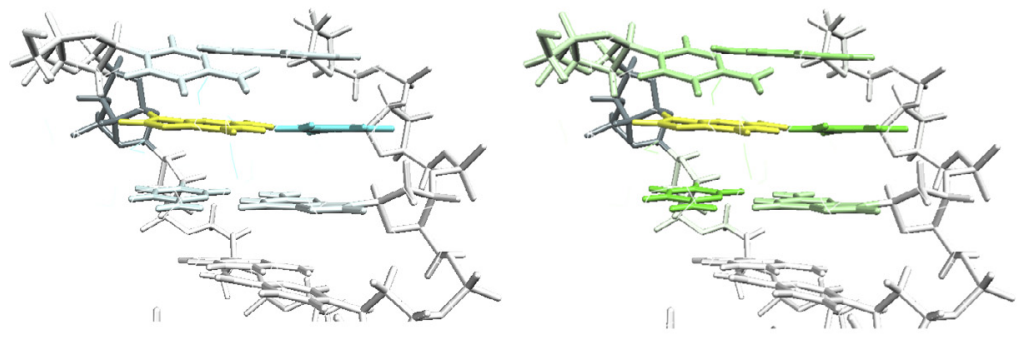

$-5.0$

0.0

$-10.0$

0.0

Main component

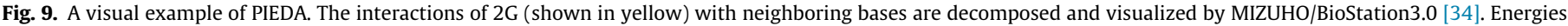
are in $\mathrm{kcal} / \mathrm{mol}$. (For interpretation of the references to color in this figure legend, the reader is referred to the web version of this article.)

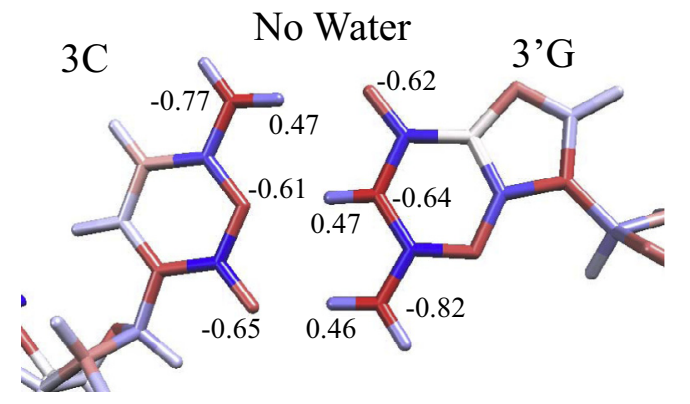

Base charge : -0.18

$-0.28$

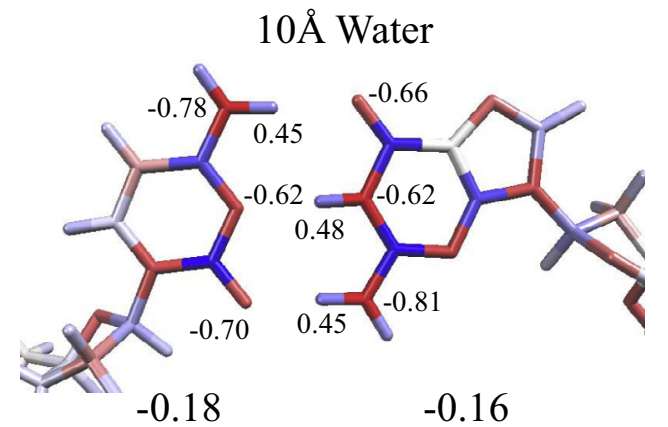

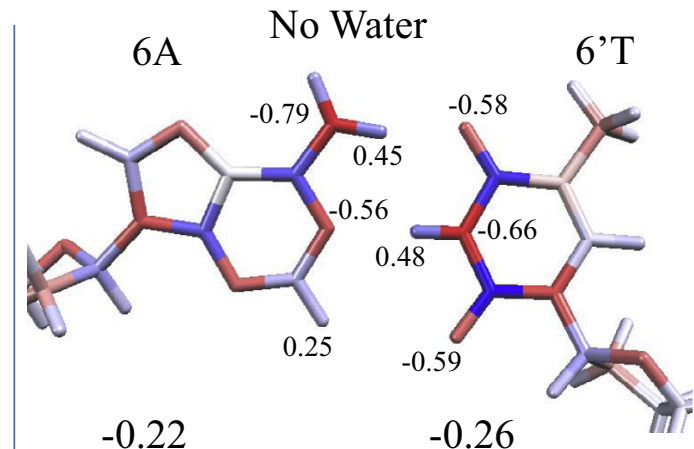

$10 \AA$ Water

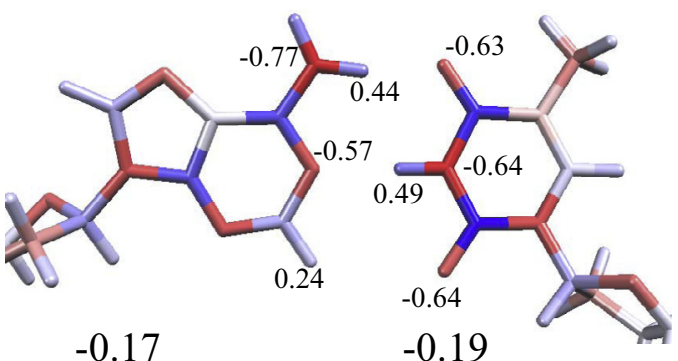

$-0.17$

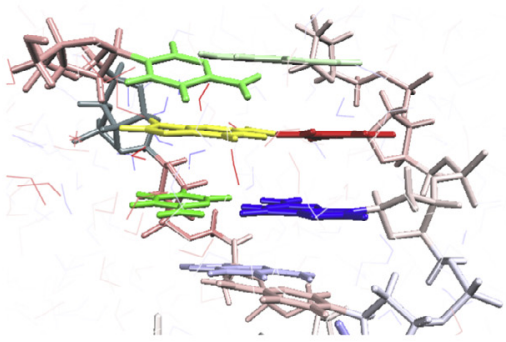

NPA/MP2 charge -1.0

$+1.0$

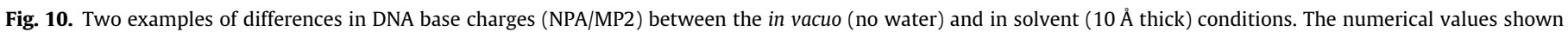
beside some of the atoms are their partial charges $(e)$ obtained via NPA.

negative and positive components, the most important stabilizing contribution was the dispersion (DI; Table Si2-2 A and B). A visual example of PIEDA is shown for the interaction of $2 \mathrm{G}$ with neighbor- ing bases (Fig. 9). Prominent stabilizing interactions with 2G were seen in $2^{\prime} \mathrm{C}$, the partner of the $\mathrm{W}-\mathrm{C} \mathrm{H}$-bond, and in $1 \mathrm{C}$ and $3 \mathrm{C}$, the partners of stacking interactions. The main components of these 
stabilizing interactions were ES for $2 \mathrm{C}^{\prime}$ and DI for $1 \mathrm{C}$ and $3 \mathrm{C}$. Nevertheless, the difference between in solvent and in vacuo consisted mostly of ES both in H-bond and stacking (Table Si2-2C), indicating that the modulation of the electrostatic interaction mainly accounts for the solvent-induced destabilization of the stacking interaction.

Thus, both the stabilization of the H-bonds and the destabilization of the stacking upon solvation were due to the modulation of the electrostatic interaction. This modulation was further analyzed by looking into the charge redistribution among bases and phosphates elicited by the solvation (Table Si2-3). As is clearly indicated by the charge difference ( $Q s-Q v)$, a significant amount of negative charge was transferred to the solvent from all the bases and phosphates of DNA. This charge loss should have resulted in relaxation of the charge-charge repulsion within DNA, thereby intensifying the $\mathrm{W}-\mathrm{C} \mathrm{H}$-bond interaction. Nevertheless, the net charge on each base and phosphate alone cannot account for the destabilization of the stacking. We therefore investigated the atomic charge distribution of the base pairs and found that the solvent induced polarization of atomic charges within the bases (see Fig. 10 for two representative base pairs). The absolute values of the net charges of the bases were smaller in solvent than in vacuo, but the atomic charges of the atom pairs forming $\mathrm{W}-\mathrm{C} \mathrm{H}$-bonds were more polarized in solvent. We speculate that this polarization intensified the $\mathrm{W}-\mathrm{C} \mathrm{H}$-bonds synergistically with the charge-charge repulsion but that it weakened the inter-strand stacking at the same time.

Based on the above discussion, the effect of solvation on DNA can be summarized as follows. Upon solvation a significant amount of negative charge is transferred from DNA to the solvent, and this charge transfer weakens the repulsion on the part of DNA and thereby strengthens the $\mathrm{W}-\mathrm{C} \mathrm{H}$-bonds. The solvation also elicits polarization within the base, and this intensifies the $\mathrm{W}-\mathrm{C}$ H-bonds but weakens the base-base stacking.

\section{Conclusion}

We have investigated the effect of an explicit solvent, i.e., water and $\mathrm{Na}^{+}$ions, on several physical properties of DNA via FMO. We found that a solvent shell as thick as $8 \AA$ is necessary to incorporate the solvent effect. Conversely, qualitative or semi-quantitative evaluation of the properties was possible by addition of an $8 \AA$ solvent shell to DNA, even without neutralization of the net charge of the target. This finding justifies quantum-chemical calculation of a solvated DNA by clipping the solvent, even if the resultant molecular configuration carries some charge.

The solvent has been shown to stabilize the $\mathrm{W}-\mathrm{C}$ H-bonding and to destabilize the stacking interactions within DNA mainly by modulation of the electrostatic interaction. Upon solvation, a charge of ca. $-0.6 e$ /base pair is transferred from DNA to the solvent. This charge transfer elicits a charge redistribution within DNA, which leads to a change in the electrostatic environment and thereby modulates the base-base interactions.

We have thus reported intensive benchmark FMO calculations of DNA together in Ref. [19] and this article. We hope that our findings will serve as guiding principles for FMO and related quantum calculations of DNA and possibly RNA, the molecules extensively studied for biological, medical, and technological purposes.

\section{Acknowledgements}

We thank Ms. Haruka Yamada for several test calculations and Ms. Mieko Nakamura for providing Fig. 8. This work was supported by funds from the following: the Core Research for Evolutional Science and Technology (CREST) project of the Japan Science and Technology Agency (JST) to ST, YM, KF and YK; a Grant-in-Aid for
Scientific Research from the Ministry of Education, Culture, Sports, Science and Technology of Japan (MEXT) to YM ("Molecular Theory for Real Systems") and to YM and YK ("Molecular-level Analyses of Dynamics-controlled Organic Reactions"); the Rikkyo University Special Fund for Research (SFR) to YM, YK and KF; and the Research and Development of Innovative Simulation Software (RISS) project at the Institute of Industrial Science of the University of Tokyo to $\mathrm{KF}, \mathrm{YM}, \mathrm{YO}$, and CW.

\section{Appendix A. Supplementary material}

Supplementary data associated with this article can be found, in the online version, at http://dx.doi.org/10.1016/j.comptc.2014.1 1.020 .

\section{References}

[1] D.L. Beveridge, T.E. Cheatham III, M. Mezei, The ABCs of molecular dynamics simulations on B-DNA, circa 2012, J. Biosci. 37 (2012) 379-397.

[2] I. Cozmuta, H. Mehrez, DNA modeling within ab initio and empirical methods, J. Comput. Theor. Nanosci. 4 (2007) 349-383.

[3] C. Alhambra, F.J. Luque, F. Gago, M. Orozco, Ab initio study of stacking interactions in A- and B-DNA, J. Phys. Chem. B 101 (1997) 3846-3853.

[4] D. Svozil, P. Hobza, J. Sponer, Comparison of intrinsic stacking energies of ten unique dinucleotide steps in A-RNA and B-DNA duplexes. Can we determine correct order of stability by quantum-chemical calculations?, J Phys. Chem. B 114 (2010) 1191-1203.

[5] L.M. Westerhoff, K.M. Merz Jr., Quantum mechanical description of the interactions between DNA and water, J. Mol. Graphics Model. 24 (2006) 440-455.

[6] F.L. Gervasio, P. Carloni, M. Parrinello, Electronic structure of wet DNA, Phys Rev. Lett. 89 (2002) 108102-1-108102-4

[7] K. Kitaura, F. Ikeo, F. Asada, T. Nakano, M. Uebayasi, Fragment molecular orbital method: an approximate computational method for large molecules, Chem. Phys. Lett. 313 (1999) 701-706.

[8] D.G. Fedorov, K. Kitaura (Eds.), The Fragment Molecular Orbital Method: Practical Applications to Large Molecular Systems, CRC Press, London, 2009.

[9] S. Tanaka, Y. Mochuzuki, Y. Komeiji, Y. Okiyama, K. Fukuzawa, Electroncorrelated fragment-molecular-orbital calculations for biomolecular and nano systems, Phys. Chem. Chem. Phys. 16 (2014) 10310-10344.

[10] T. Nakano, T. Kaminum, T. Sato, K. Fukuzawa, Y. Akiyama, M. Uebayasi, K. Kitaura, Fragment molecular orbital method: use of approximate electrostatic potential, Chem. Phys. Lett. 351 (2002) 475-480.

[11] S. Amari, M. Aizawa, J. Zhang, K. Fukuzawa, Y. Mochizuki, Y. Iwasawa, K. Nakata, H. Chuman, T. Nakano, VISCANA: visualized cluster analysis of protein-ligand interaction based on the ab initio fragment molecular orbital method for virtual ligand screening. J. Chem. Inf. Model. 46 (2006) 221-230.

[12] I. Kurisaki, K. Fukuzawa, Y. Komeiji, Y. Mochizuki, T. Nakano, J. Imada, A. Chmielewski, S.M. Rothstein, H. Watanabe, S. Tanaka, Visualization analysis of inter-fragment interaction energies of CRP-CAMP-DNA complex based on the fragment molecular orbital method, Biophys. Chem. 130 (2007) 1-9.

[13] D.G. Fedorov, K. Kitaura, Pair interaction energy decomposition analysis, J. Comput. Chem. 28 (2007) 222-237.

[14] T. Ishikawa, Y. Mochizuki, T. Nakano, S. Amari, H. Mori, H. Honda, T. Fujita, H. Tokiwa, S. Tanaka, Y. Komeiji, K. Fukuzawa, K. Tanaka, E. Miyoshi, Fragment molecular orbital calculations on large scale systems containing heavy metal atom, Chem. Phys. Lett. 427 (2006) 159-165.

[15] K. Fukuzawa, Y. Komeiji, Y. Mochizuki, A. Kato, T. Nakano, S. Tanaka, Intra- and intermolecular interactions between cyclic-AMP receptor protein and DNA: ab initio fragment molecular orbital study, J. Comput. Chem. 27 (2006) 948-960.

[16] T. Watanabe, Y. Inadomi, K. Fukuzawa, T. Nakano, S. Tanaka, L. Nilsson, U. Nagashima, DNA and estrogen receptor interaction revealed by fragment molecular orbital calculations, J. Phys. Chem. B 111 (2007) 9621-9627.

[17] T. Watanabe, Y. Inadomi, H. Umeda, K. Fukuzawa, S. Tanaka, T. Nakano, U. Nagashima, Fragment molecular orbital (FMO) and FMO-MO calculations of DNA: accuracy validation of energy and interfragment interaction energy, J. Comput. Theor. Nanosci. 6 (2009) 1328-1337.

[18] I. Kurisaki, K. Fukuzawa, T. Nakano, Y. Mochizuki, H. Watanabe, S. Tanaka, Fragment molecular orbital (FMO) study on stabilization mechanism of neurooncological ventral antigen (NOVA)-RNA complex system, J. Mol. Struct: THEOCHEM 962 (2010) 45-55.

[19] K. Fukuzawa, C. Watanabe, N. Taguchi, Y. Mochizuki, T. Nakano, S. Tanaka, Y. Komeiji, Accuracy of the fragment molecular orbital (FMO) calculations for DNA: total energy, molecular orbital, and inter-fragment interaction energy, Comput. Theor. Chem. 1034 (2014) 7-16.

[20] Y. Komeiji, T. Ishida, D.G. Fedorov, K. Kitaura, Change in a protein's electronic structure induced by an explicit solvent: an ab initio fragment molecular orbital study of ubiquitin, J. Comput. Chem. 28 (2007) 1750-1762.

[21] X. Shui, L. McFail-Isom, G.G. Hu, L.D. Williams, The B-DNA dodecamer at high resolution reveals a spine of water on sodium, Biochemistry 37 (1998) 83418355. 
[22] Y. Komeiji, M. Uebayasi, R. Takata, A. Shimizu, T. Itsukashi, M. Taiji, Fast and accurate molecular dynamics simulation of a protein using a special purpose computer, J. Comput. Chem. 18 (1997) 1546-1563.

[23] Y. Komeiji, M. Haraguchi, U. Nagashima, Parallel molecular dynamics simulation of a protein, Parallel Comput. 27 (2001) 977-987.

[24] J. Wang, P. Cieplak, P.A. Kollman, How well does a restrained electrostatic potential (RESP) model perform in calculating conformational energies of organic and biological molecules?. J Comput. Chem. 21 (2000) 1049-1074.

[25] W.L. Jorgensen, J. Chandrasekhar, J.D. Madura, R.W. Impey, M.L. Klein, Comparison of simple potential functions for simulating liquid water, J. Chem. Phys. 79 (1983) 926-935.

[26] X.-J. Lu, W.K. Olson, 3DNA: a software package for the analysis, rebuilding and visualization of three-dimensional nucleic acid structures, Nucl. Acids Res. 31 (2003) 5108-5121.

[27] T. Nakano, T. Kaminuma, T. Sato, Y. Akiyama, M. Uebayasi, K. Kitaura, Fragmen molecular orbital method: application to polypeptides, Chem. Phys. Lett. 318 (2000) 614-618.

[28] ABINIT-MP 6.0 and BioStation Viewer 15.00, Research and development of innovative simulation software, 2013 <http://www.ciss.iis.u-tokyo.ac.jp/ software/>.

[29] Y. Mochizuki, T. Nakano, S. Koikegami, S. Tanmori, Y. Abe, U. Nagashima, K. Kitaura, A parallelized integral-direct second-order Moeller-Plesset perturbation theory method with a fragment molecular orbital scheme, Theor. Chem. Acc. 112 (2004) 442-452.
[30] Y. Mochizuki, S. Koikegami, T. Nakano, S. Amari, K. Kitaura, Large scale MP2 calculations with fragment molecular orbital scheme, Chem. Phys. Lett. 396 (2004) 473-479.

[31] Y. Mochizuki, K. Yamashita, T. Murase, T. Nakano, K. Fukuzawa, K. Takematsu, H. Watanabe, S. Tanaka, Large scale FMO-MP2 calculations on a massively parallel-vector computer, Chem. Phys. Lett. 457 (2008) 396-403.

[32] A.E. Reed, F. Weinhold, Natural bond orbital analysis of Near-Hartree-Fock water dimer, J. Chem. Phys. 78 (1983) 4066-4073.

[33] A.E. Reed, R.B. Weinstock, F. Weinhold, Natural population analysis, J. Chem. Phys. 83 (1985) 735-746.

[34] MIZUHO/BioStation3.0, Mizuho information and research institute Inc, 2013.

[35] F. Jensen, Introduction to Computational Chemistry, second ed., Wiley \& Sons, West Sussex, 2007.

[36] T. Fujita, K. Fukuzawa, Y. Mochizuki, T. Nakano, S. Tanaka, Accuracy of fragmentation in ab initio calculations of hydrated sodium cation, Chem. Phys. Lett. 478 (2009) 295-300.

[37] T. Fujiwara, Y. Mochizukia, Y. Komeiji, Y. Okiyama, H. Mori, T. Nakano, E. Miyoshi, Fragment molecular orbital-based molecular dynamics (FMO-MD) simulations on hydrated Zn(II) ion, Chem. Phys. Lett. 490 (2010) 41-45.

[38] H. Sugiyama, I. Saito, Theoretical studies of GG-specific photocleavage of DNA via electron transfer: significant lowering of ionization potential and 5'localization of HOMO of stacked GG bases in B-form DNA, J. Am. Chem. Soc. 118 (1996) 7063-7068. 